\title{
¿PRACTICAMOS LO QUE PREDICAMOS?
}

\author{
JAMES A. F. STONER \\ Escuela de Negocios Gabelli \\ Universidad de Fordham, Nueva York, Nueva York, EEUU \\ stoner@fordham.edu
}

En el primer número de esta revista, William L. Weis, profesor de la Universidad de Seattle, preguntó suavemente sobre la medida en que estábamos practicando lo que predicábamos con respecto a la sostenibilidad en su propia escuela así como en las otras escuelas de negocios jesuitas (Weis, 2013). Quizás puedes decir que estaba reprochándonos la falta de acciones al respecto. Tanto la naturaleza de su investigación como las preguntas son universales, aplicables a todas las escuelas de negocios y universidades de todo el mundo, pero los desafíos que él presenta son especialmente apropiados a las escuelas de negocios jesuitas (y universidades) debido a sus valores basados en la fe y declaraciones de misión explícitas.

Siete años después, Marinilka Barros Kimbro, Rubina Mahsud y David Adut, de la Escuela de Negocios y Empresariales de Albers de la Universidad de Seattle, continúan este tipo de investigación y la llevan a un nuevo nivel preguntando a las universidades jesuitas si están demostrando lo que dicen en cuanto a la inversión de los fondos de dotación. En el primero de dos artículos en este número, exploran hasta qué punto las universidades jesuitas en los Estados Unidos están apoyando la industria de fósiles combustibles que contribuye tanto al cambio climático y al calentamiento global que destruyen rápidamente la capacidad del planeta para mantener a nuestra especie y también las de otras criaturas. $\mathrm{O}$, más precisamente, exploran hasta qué punto esas instituciones se han desinvertido de las empresas de combustibles fósiles a través de la eliminación de acciones de combustibles fósiles de sus carteras de dotación.

Luego, en el siguiente artículo, Kimbro, Mahsud y Adut continúan a estudiar las razones que con frecuencia se ofrecen a favor o en contra de la desinversión de valores de combustibles fósiles. Descubren que, al final, las razones a favor de la desinversión son más fuertes que las que están en contra, y llegan a la conclusión de que todas las universidades, jesuitas o no, deberían desinvertir de tales valores no 
solo por razones morales y éticas, sino también por motivos financieros. Razonan que desinvertir de una industria cuyo futuro está cada vez más en duda puede ser más prudente que continuar teniendo valores. Por lo tanto, se puede alinear las responsabilidades fiduciarias y morales de los oficiales de inversión de la dotación de la universidad morales si desinvierten ahora.

Estos artículos de Weis, Kimbro, Mahsud y Adut, así como los otros cuatro en este número, nos recuerdan que necesitaremos buscar respuestas a cinco preguntas principales, y tomar medidas sobre ellos si queremos encontrar alguna forma de enfrentarnos al mayor desafío intelectual y moral que nuestra especie ha tenido.

1. ¿Cómo podemos lidiar, muy muy pronto, con el cambio climático, el calentamiento global y sus efectos inmediatos (el descubrimiento y la acción)?

2. ¿Qué tipo de personas necesitaremos ser para florecer en este planeta sin destruirlo, e incluso, curarlo (el descubrimiento)?

3. ¿Cómo podemos, todos nosotros, convertirnos en ese tipo de persona, con dignidad, respeto y cuidado por circunstancias únicas y formas de ser muy muy rápidamente (la acción)?

4. ¿Qué tipos de producción, distribución y sistemas de consumo que protegen el planeta proporcionarán los bienes y servicios necesarios para que todos podamos llevar una vida floreciente (el descubrimiento)?

5. ¿Cómo podemos crear esos sistemas (la acción)?

El descubrimiento y la acción por el bien común siempre han sido la misión fundamental de la educación empresarial. Sin embargo, esa misión se perdió hace mucho tiempo en la definición simplista y equivocada del primer y principal (y últimamente, el "único") objetivo de la empresa, que es enriquecer a los accionistas, los ejecutivos y los comerciantes especulativos. Es una definición justificada, en parte, por las afirmaciones de que la búsqueda desenfrenada de fines egoístas garantiza el mayor nivel del bien común y que somos cascarones vacíos, tanto moralmente como emocionalmente, buscando solo un consumo cada vez mayor e interminable 
para lograr lo que percibimos que es la verdadera felicidad y los deseos de nuestros corazones. Tales perspectivas han sido reforzadas por una insistencia incuestionable de que la aceptación de la narrativa neoliberal por sí sola nos protegerá de movernos, como leminos, en el camino hacia la servidumbre, algo inevitable en sociedades donde cualquier aspecto de la voluntad popular se expresa a través de alguna forma de acción gubernamental.

Por lo tanto, enmarcar estas cinco preguntas en términos de descubrimiento y acción puede sugerir oportunidades que las escuelas de negocios de todo el mundo tienen para desviar un curso de "bajíos peligrosos y cachones tremendos" ("dangerous shoals and roaring breakers") (Stobaugh, 2012) ${ }^{1}$ del mundo. Por fin, nos estamos dando cuenta de que nos hemos estado moviendo despreocupadamente a esos bajíos y cachones por más de medio siglo. Estas instituciones pueden desempeñar un papel muy importante tanto en comprender las acciones necesarias para alejarnos de nuestra dependencia de los combustibles fósiles y en provocar ese cambio muy rápidamente. Pueden tomar la iniciativa en responder a las cinco preguntas o desafíos arriba mencionados, y en buscar alternativas al paradigma neoliberal dominante, en particular, como las que se sugieren en el ensayo invitado escrito por el Padre Garanzini en este número. Estos desafíos están hechos a medida para el liderazgo y contribución de las escuelas de negocios, instituciones que tienen misiones, recursos intelectuales y financieros (sí, incluso en este periodo de agitación inducida por el coronavirus y la incertidumbre), e incluso el interés propio para descubrir y dar vida a las respuestas y acciones que necesitamos.

Por supuesto, hay muchos problemas y tragedias relacionados con la justicia social, la pobreza global, el bienestar, la dignidad humana, la moralidad, la ética y el cuidado que requieren nuestra atención todos los días. Algunos están muy conectados con estos cinco "problemas" o "desafíos," y otros no. Cuando hayamos solucionado el problema inmediato de la actual pandemia mundial de la COVID-19, cuando nos hayamos preparado (con suerte) para la próxima, sea lo que sea, y cuando hayamos "resuelto" el problema urgente del cambio climático que permite que nuestra especie permanezca en este planeta y florezca, todavía tendremos que lidiar con una infinidad de otras preocupaciones: el VIH/SIDA, el hambre, enfermedades aparentemente interminables, la bomba demográfica que explotó hace mucho tiempo, la extinción de especies, etc. Sin embargo, no estaremos aquí 
para enfrentarnos a estos otros problemas si no "resolvemos" el problema del cambio climático. En ese sentido, lidiar con el cambio climático y el calentamiento global es nuestra "primera prioridad."

\section{No hay VIH/SIDA, ni hambre, ni injusticia social en un planeta muerto.}

Entonces, para las escuelas de negocios, ¿ahora, qué? Los otros artículos en este número continúan los intentos de abordar partes de la pregunta "¿y ahora, qué?" que Weis, Kimbro, Mahud y Adut han contestado.

En el ensayo invitado que aparece primero en este número, el Padre Michael Garanzini nos informa sobre una consulta con respecto al propósito y el papel de la educación empresarial jesuita que puede ir mucho más allá de lo que se enseña y se investiga en las escuelas de negocios que son "solo" jesuitas o incluso "solo" católicos. Esa consulta es parte de un reconocimiento mundial creciente de que tanto la educación empresarial estadounidense como la global, cada vez más, por al menos medio siglo, han sido servidores activos y facilitadores de las prácticas de la mentalidad de "negocio como siempre" que han estado destruyendo la capacidad del planeta para apoyar nuestra especie y la de otras. Como lo notan Laszlo, Sroufe, and Waddock (2017), la educación empresarial ha sido, en gran medida y con algunas excepciones notables y valiosas, tanto la víctima como el siervo de la narrativa neoliberal que ha inculcado en nosotros las mentalidades, valores, herramientas y weltanschaaung de "negocio como siempre" que han jugado un papel importante en meternos en el lío global en el que estamos ahora.

Por lo tanto, si bien lo que el P. Garanzini informa es dirigido desde y elaborado en términos de valores y misiones jesuitas y católicas romanas, es mucho más que un llamado a un paradigma inspirado de la educación empresarial jesuita dirigida a los jesuitas y romanos instituciones católicas. Será recibido por muchos otros como algo mucho más que un simple intento para "construir la marca jesuita" o mejorar la posición de las escuelas de negocios jesuitas en la competencia para estudiantes y recursos. Será recibido por muchos como lo que se pretende ser, una invitación en el espíritu de Laudato Si' para todos los educadores y líderes de negocios (profesores, estudiantes, ex alumnos y simpatizantes) para dialogar sobre lo que la educación empresarial puede llegar a ser para el bien común. Y, más importante, es una invitación extendida, a modo de ejemplo, para motivar a las escuelas de negocio 
a llevar a cabo sus propios diálogos sobre las acciones que tenemos que tomar para hacer frente a los desafíos que enfrentamos.

En su artículo "La evaluación de iniciativas de sostenibilidad en las instituciones de educación superior," Justin Edric G. Yturzaeta de la Universidad Ateneo de Manila se centra directamente en la tarea de asegurarse de que la educación en las escuelas de negocios y en otras instituciones esté realmente orientada hacia un mundo sostenible, floreciente y regenerador. Observa que cada una de las instituciones de educación superior desempeña un papel único en el panorama académico dado su contexto especial, a saber, su ubicación geográfica, sus recursos, el mercado objetivo y las áreas de especialización. Sin embargo, todas esas instituciones se enfrentan al desafío de centrar el desarrollo no sólo en su investigación y currículo, sino también en sus operaciones como organizaciones.

Yturzaeta describe cómo se ha desarrollado una herramienta rápida de evaluación de la sostenibilidad para las instituciones de educación superior a fin de ayudarles a evaluar y gestionar sus iniciativas de sostenibilidad. La herramienta también actúa como un marco que puede ayudar a las instituciones de educación superior a maximizar el valor sostenible que crean en sus propios contextos ahora y en el futuro, dentro y fuera de su organización, y en las dimensiones ambientales, sociales, gubernamentales y académicas. En el espíritu, entonces, del lema de negocios citado a menudo, "lo que se mide, se hace," el estudio de Yturzaeta ofrece los medios para ayudar a las escuelas de negocios y otras instituciones a hacer parte del trabajo de sostenibilidad, regeneración y florecimiento global.

En el artículo "Creando un negocio sostenible: ¿Cómo se sucede?: Una exploración de motivadores y facilitadores en tres entornos organizativos en los EEUU," Robin T. Byerly de la Universidad Estatal de Appalachia, aborda las palabras cuarta y quinta arriba mencionadas para la educación de empresariales, en particular con respecto a cómo podemos crear los tipos de organizaciones productivas que necesitamos. Ella empieza reconociendo la premisa fundamental de que los esfuerzos para gestionar la sostenibilidad deben extenderse a todas las organizaciones e instituciones, ya sean pequeñas, grandes, nacionales o globales. Señala que, si bien muchas empresas ya participan en iniciativas de sostenibilidad, más honestas y expansivas que otras, muchas no están motivadas o no trabajan para la sostenibilidad en sus modelos de negocio. Las empresas de Interface Carpet y Patagonia son algunos 
ejemplos notables de empresas que han aceptado plenamente la dedicación de ser sostenibles en todos los aspectos de la operación. Sin embargo, es cada vez más necesario que más empresas lo hagan en la mayor medida posible.

Byerly pregunta, por lo tanto, cómo podemos entender mejor las formas en que varias instituciones realmente crean modelos de sostenibilidad, observando que la teoría proporciona factores y circunstancias motivadores que podrían impedir que las empresas intenten avanzar hacia un mayor grado de sostenibilidad. Su artículo presenta tres casos organizativos de empresas que son parecidas en su propósito, búsqueda y singularidad declarados de sostenibilidad, y sin embargo, son diferentes en tamaño, industria y ambiente. Si bien estas diferencias son significativas como se puede ver en la evidencia triangulada que presenta, se puede también aprender lecciones de muchas similitudes que surgen a pesar de las diferencias. Es cierto que todavía hay mucho que aprender de la exploración de instituciones que participan activamente en estos esfuerzos y de la investigación profunda de las realidades motivadoras y facilitadoras que representan.

En el artículo "¿Startup o scaleup? Un enfoque a través del impacto económico," Joaquín García-Tapial y Manuel Alejandro Cardenete de la Universidad Loyola Andalucía exploran la cuestión muy importante de la escalabilidad para las iniciativas y organizaciones de sostenibilidad. Distinguen entre las organizaciones emergentes como instituciones en sí mismo y las que crecen rápidamente, y observan que si bien el espíritu emprendedor ha sido considerado tradicionalmente como uno de los principales catalizadores de la economía (y por lo tanto, de desarrollo sostenible también), no fue hasta hace varios años que las autoridades públicas de varias partes del mundo planearon y organizaron esfuerzos para apoyar iniciativas empresariales.

Sin embargo, la eficacia de estas inversiones, que alcanzan hasta millones de euros en un año, rara vez se considera en relación con el impacto que tiene tal actividad empresarial en la economía. No obstante, en los últimos meses, las autoridades públicas se han centrado más en las llamadas scaleups, es decir, las startups que han experimentado un crecimiento de más de $20 \%$ en un periodo de al menos tres años consecutivos. La creencia general, a pesar del hecho de que sólo se han elaborado unos pocos estudios sobre este fenómeno, es que estas empresas tienen un impacto considerable en la economía, especialmente en términos de empleo. 
Según García-Tapial y Cardenete, debemos preguntarnos si tenemos que seguir dedicando escasos recursos públicos a la generación de nuevas empresas (startups) o redirigirlos hacia la promoción de scaleups. El objetivo principal de su trabajo consiste en explorar y examinar si las autoridades deben cambiar el destino de recursos, es decir, en lugar de apoyar a las startups, deben fomentar las scaleups, según su impacto económico. Estudian el impacto económico de la actividad empresarial (startups) y de un tipo particular de empresa de alto crecimiento en las que las autoridades públicas ahora están centrándose (scaleups) y comparan los impactos de ambos para sacar conclusiones. El estudio se centra en Andalucía, una de las regiones con los niveles más bajos de desarrollo en la Unión Europea.

¿Y ahora, qué más?

\section{CONTINUANDO LA EXPLORACIÓN DE LA DESINVERSIÓN DE COMBUSTIBLES FÓSILES EN EL ESPÍRITU DE LAUDATO SI'}

Los autores en este número son partidarios fervientes de las acciones que quieren que hagamos. Sus trabajos nos llaman a participar en los tipos de diálogo que el Papa Francisco nos invita a nosotros, y a toda la comunidad mundial, a hacer mientras luchamos contra los asuntos y desafíos muy grandes de la sostenibilidad y la justicia social que él y los muchos asesores expertos e instituciones que apoyaron su investigación y redacción identifican en Laudato Si' (Francisco, 2015). Diálogos verdaderos, en el espíritu y la práctica de la investigación humilde que Ed Schein (2013) describe con tanta gracia, pueden ser formas poderosos pero suaves de crear los compromisos que debemos asumir, los compromisos que son necesarios para llevar a cabo el arduo trabajo que todos debemos hacer.

Entonces, en el espíritu de tales diálogos, sugerimos que se celebre una conferencia virtual el 10 y el 11 de junio de 2021, jueves y viernes, aproximadamente un mes antes del $26^{\circ}$ Foro Mundial Anual de la Asociación Internacional de Escuelas de Negocios Jesuitas y la $22^{a}$ Reunión Anual de los Colegas de la Educación Empresarial Jesuita en ITESO, en Tlaquepaque, Jalisco, México, y que se invite a los directores financieros responsables de invertir las dotaciones de cada una de las 27 universidades jesuitas en los Estados Unidos. Si bien los detalles exactos de la conferencia seguramente se cambiarán con el tiempo, nuestra sugerencia preliminar es que las sesiones se reúnan desde el mediodía a las siete de la noche, hora del 
este (de 9 a 4, tiempo del Pacífico) durante los dos días mencionados, con café y pausas programados. Se pedirá a cada uno de los 27 oficiales de las universidades jesuitas que den un resumen introductorio muy corto (p.ej. de cinco minutos más o menos) de dónde están ahora en cuanto al asunto de desinversión de empresas de combustibles fósiles, qué planes tienen en el futuro sobre este tema, y qué podría ser más útil para ellos cuando consideren la cuestión de la desinversión o no de empresas de combustibles fósiles. En cuanto a algunos que ya se han desinvertido, por supuesto, pueden informar sobre el razonamiento que los llevó a desinvertir, y cómo se fueron a hacerlo.

La mayor parte del tiempo de la conferencia virtual se dedicaría al diálogo abierto en el espíritu de Laudato Si', celebrado en la forma clásica: no juicioso y con confidencia abierta, y de acuerdo con las directrices para el diálogo presentadas por Walter Isaacs (1999) y otros. El diseño sugerido para la conferencia no incluiría compromisos ni entendimientos formales, en lugar de colocar los documentos de postura antes de las reuniones o el comunicado del fin de la conferencia, sólo habría pensamientos compartidos, preguntas, y tal vez sueños y esperanzas. Se podría poner las conversaciones de la conferencia a disposición del público a medida que se produzcan, y se podría archivar las sesiones podrían para el acceso futuro de cualquier persona interesada.

Una de las lecciones aprendidas de las tragedias de la COVID-19, que se estaban ocurriendo mientras que este editorial se escribía, es que podemos llevar a cabo una conferencia sin costo utilizando las tecnologías disponibles hoy en día. Además, asistir a ella no dará ningún sentimiento de "culpabilidad" por el uso del carbono ya que nadie tiene que viajar a un sitio común de conferencias. El internet nos unirá con unos pocos clics y sin ninguna carga sobre los presupuestos universitarios. Para los que puedan estar interesados, se puede contactar directamente con los organizadores de la conferencia a través de kimbrom@seattleu.edu y stoner@globalmovement.net. 


\section{BIBLIOGRAFÍA}

Francisco. 2015. Laudato si': Sobre el cuidado de la casa común. Ciudad de Vaticano: Libreria Editrice Vaticana.

Isaacs, W. 1999. Dialogue and the art of thinking together: A pioneering approach to communicating in business and in life. Nueva York: Currency/Doubleday.

Laszlo, C., Sroufe, R., \& Waddock, S. 2017. Torn between two paradigms: A struggle for the soul of business schools. AI Practitioner, 19(2): 108-119.

Philbrick, N. 2007. Mayflower: Voyage, community, war. Nueva York: Penguin

Schein, E. H. 2013. Humble inquiry: The gentle art of asking instead of telling. San Francisco: Berrett-Koehler.

Stobaugh, J. P. 2012. World literature: Cultural influences of early to contemporary voices. Green Forest, AR: New Leaf Publishing Group.

Weis, W. L. 2013. Hypocrisy at the lectern: Do our personal lifestyle choices reflect our spoken commitment to global sustainability? Journal of Management for Global Sustainability, 1(1): 29-45. 